\title{
DIVERSIDAD COMO ESTRATEGIA DE PROYECTO EN LA TRANSFORMACIÓN URBANA
}

\author{
Anamaria DE ARAGÃO C. MARTINS
}

El proceso de deslocalización de la segunda mitad del siglo xx y la reflexión acerca de la utilización de nuevos mecanismos de planeamiento, proyecto y gestión urbana en la ciudad consolidada crearon la oportunidad de intervenciones de larga escala en las periferias industriales históricas. El proyecto de transformación urbana responde a las especificidades de este territorio entrecortado por obstáculos de diferente naturaleza y contaminado por variadas preexistencias de su pasado industrial, creando nuevas actividades y nuevas oportunidades para barrios periféricos. Basado en la tesis de doctorado desarrollada sobre diferentes intervenciones urbanas, el presente artículo estudia las estrategias de diversidad utilizadas en los proyectos de transformación urbana en la definición de los trazados y en la composición de los volúmenes edificados para evaluar el encaje del nuevo fragmento urbano en su contexto.

Transformación urbana, proyecto urbano, periferias industriales.

\section{DIVERSITY AS A PROJECT STRATEGY IN URBAN TRANSFORMATION.}

The process of industry relocation of the second half of the twentieth century and the reflection on the use of new mechanisms for planning, design and urban management in the consolidated city, created an opportunity to large scale interventions in the historical industrial peripheries. The urban transformation project responds to the specificity of this territory, interrupted by obstacles of different kinds and pre-existing conditions by its industrial past, and creating new activities gives new opportunities for suburbs. The present article examines the different strategies used, defining paths and the composition of the built volumes in urban transformation projects to evaluate the fit the new urban fragment in their context.

Urban transformation, urban project, industrial peripheries. 
El fenómeno de la transformación urbana de los últimos 25 años ha operado sobre las áreas industriales, ferroviarias y portuarias de las periferias históricas, como consecuencia del proceso de deslocalización industrial de la segunda mitad del siglo $\mathrm{xx}$.

Las actividades industriales buscaron nuevos sitios, más distantes del centro, donde podrían adquirir terrenos de mayor dimensión y menor valor (DIPASQUALE \& WHEATON, 1996). La amplia red viaria, consolidada en la segunda mitad del siglo $\mathrm{xx}$, ha permitido mayor o igual accesibilidad de las nuevas áreas industriales, en comparación con las antiguas. Así, recintos industriales obsoletos y terrenos abandonados han proliferado en las antiguas periferias.

El proceso de degradación ha abierto la oportunidad de intervención en las periferias, coincidente con un momento de reflexión acerca de la utilización de nuevos mecanismos de planeamiento, proyecto y gestión urbana que buscaban dar mejor uso a la ciudad consolidada, actuando de forma diferente a los procedimientos de tabulas rasas de las décadas anteriores.

Dichos mecanismos delimitaban los fragmentos urbanos bajo intervención, resguardando el entorno inmediato al área de proyecto, como, por ejemplo, las Zones d'Aménagement Concerté-ZACs, en el caso francés, o los Planes de Reforma Interior-PERI, en Barcelona.

Durante la década de 1980, se utilizaron tales mecanismos para reformular fragmentos de la ciudad central con vistas a la estructuración de una red de espacios libres. En la década siguiente, las intervenciones se dirigieron hacia áreas más extensas de las periferias industriales, aportando nueva variable a la transformación por fragmentos: creciendo la dimensión de los terrenos de intervención, pasa a existir el riesgo de un proyecto urbano indiferente al entorno y al territorio, configurándose como una estructura urbana autónoma.

Este artículo está basado en la tesis doctoral que investiga proyectos de transformación urbana de periferias históricas en los últimos 30 años: los proyectos urbanos de Tolbiac y Masséna de la operación Paris Rive Gauche; los proyectos del Abattoirterrein, de Entrepôt-West, de Borneo y de Sporenburg en las Docas Oeste en Ámsterdam; los proyectos de la Villa Olímpica, de Diagonal Mar, de la reformulación de la Avenida Diagonal y del Frente Marítimo en el Poblenou de Barcelona y el proyecto para la Ensenada de Gamboa, en Río de Janeiro (MARTINS, 2004). 
La investigación analizó los mecanismos y los efectos del proyecto de transformación urbana, desde las estrategias de configuración de la estructura, ordenación y composición tridimensional de la forma urbana, para discutir el rol de los mecanismos de diseńo en contribuir para el buen encaje de nuevos fragmentos en el tejido urbano existente.

El análisis de la estructura del proyecto buscó entender el soporte que se establece para la forma urbana, que definirá el rol de los trazados y la naturaleza de las unidades urbanas, en parte como respuesta a los diferentes condicionantes de la transformación urbana. El análisis de la ordenación se centró en las reglas de distribución de los elementos edificados y espacios libres en las unidades urbanas, mientras que en la exploración sobre los mecanismos de composición tridimensional se indagó sobre las estrategias que definen la relación entre planos verticales y horizontales de la unidad urbana.

El presente artículo enfoca las estrategias de diversidad utilizadas en el proyecto de transformación urbana. La diversidad se presenta como un mecanismo de coherencia con territorios con gran heterogeneidad, como las primeras periferias. Además, la diversidad como mecanismo de proyecto aporta efectos que funden las preocupaciones con el paisaje urbano características de los siglos XIX y Xx, creando una nueva tendencia híbrida de composición tridimensional. Este artículo presenta la importancia de dichas estrategias de diseño urbano en obtener un buen encaje del nuevo fragmento urbano en su contexto.

\section{Las primeras periferias como espacios heterogéneos}

A medida en que crece la ciudad se forman nuevas coronas periféricas. En las aglomeraciones actuales, lo que consideramos como las "periferias" por excelencia son, de hecho, las terceras o cuartas coronas periféricas de la ciudad. Las sucesivas periferias se van alejando del centro urbano, tanto en términos de distancia, como en términos de continuidad edificada. A partir de la segunda corona periférica, los conjuntos habitacionales, polígonos de viviendas o polaridades urbanas, como centros comerciales, parques industriales, áreas de recreación, configuran un tipo de periferia caracterizada por paquetes urbanos aislados y autocontenidos, un tipo de crecimiento disperso relacionado con las infraestructuras, ausente de estructura y continuidad (MARTINS, 2004).

Las primeras periferias urbanas, que se formaron a partir de mediados del siglo XIX, por el contrario, no son carentes de estructura y continuidad. Estas periferias, dichas históricas, han crecido a lo largo de los enlaces existentes con el centro urbano y alrededor de las infraestructuras y de los recintos industriales puntuales. Con la consolidación de estos barrios de pasado in- 
dustrial, los elementos que estructuraron el crecimiento se han convertido en obstáculos urbanos, al impedir el contacto entre los tejidos urbanos adyacentes, definiendo estas periferias como territorios segregados y altamente heterogéneos (Fig. 1).
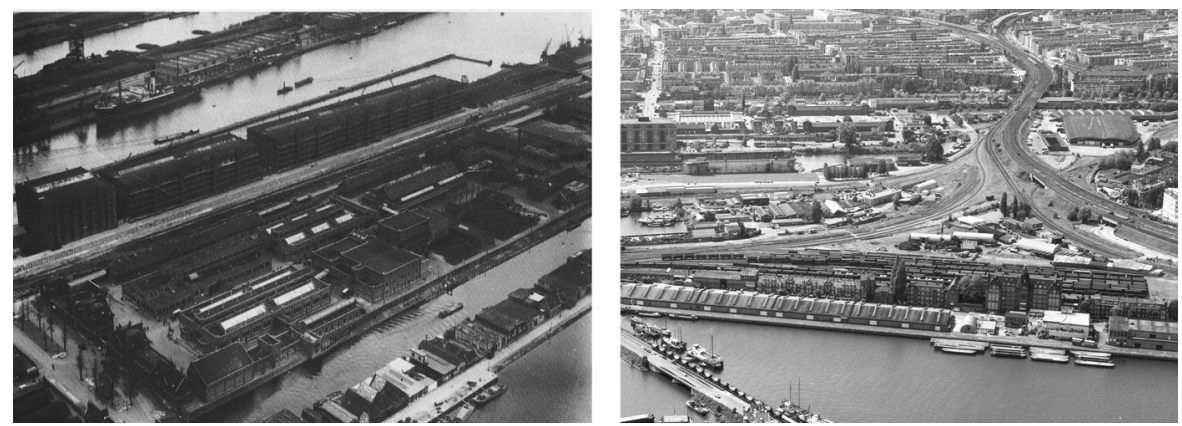

Fig.1- Docas leste de Ámsterdam en 1931(Abbatoirterrein) y 1974 (Rietlanden). fuente: KOSTER, Egbert. 1995. Het Oostelijk Havengebied Amsterdam (Eastern Docklands). Amsterdam, Architectura \& Natura, p. 34.

Rietlanden. Fuente: Gemeente Amsterdam, Dienst Ruimtelijke Ordening. G33-030, 30 juni 1986.

Se observan diferentes tipos de heterogeneidad espacial en las periferias históricas. En algunos casos, la heterogeneidad es el resultado del encuentro de las tramas de diferentes planes de expansión con los tejidos embrionarios del área. En otros, la heterogeneidad deriva de la interrupción del tejido por la presencia de obstáculos, sea un gran recinto industrial, un terminal ferroviario, instalaciones portuarias, viaductos $u$ otros tipos de infraestructura.

La heterogeneidad y la fragmentación de los tejidos de las periferias históricas determinan importante variable a ser considerada en el proyecto de transformación urbana.

\section{El proyecto urbano de finales del siglo XX: ordenaciones híbridas}

El proceso de elaboración de un proyecto urbano pasa por diferentes niveles de aproximación en la definición del espacio. En términos metodológicos, estas etapas pueden ser simultáneas o secuenciales. No obstante, en cada etapa es posible entender qué elementos se definen en cada nivel de aproximación y en base a qué criterios de diseño. El reconocimiento del nivel de detalle que cada etapa alcanza en la definición de la forma urbana permite establecer su correspondencia con determinado instrumento de planeamiento y gestión, además de los objetivos de preconfiguración de la forma urbana.

El primer nivel de aproximación del proyecto urbano es el que trata de la estructura del espacio urbano. Es la etapa en que se define el soporte que 
configura los trazados. Desde el vacío, organiza la forma urbana, es decir el espacio edificado y los espacios libres que tienen función estructurante. Su función es contribuir para el efecto del nuevo fragmento en su vinculación o independencia con relación al entorno.

El siguiente nivel de aproximación se refiere a los mecanismos de ordenación. Estos mecanismos precisan las reglas según las cuáles se distribuyen los elementos edificados sobre la unidad urbana definida por el soporte. El orden establecido define cómo se organizan los espacios libres en el interior de la manzana.

El último nivel de aproximación se refiere a la tridimensionalidad de la forma urbana. La composición de los volúmenes edificados especifica los efectos de uniformidad o diversidad presentes en cada unidad urbana y cómo estos influencian la regularidad o la irregularidad del paisaje urbano.

En la búsqueda por la diversidad, analizando los casos de estudio, fue posible constatar modelos urbanísticos que fusionan las preocupaciones presentes en el proyecto urbano de los siglos xix y en el primer tercio del siglo xx.

En la ciudad del siglo xIx los elementos fundamentales son el trazado viario, la ordenanza edificatoria y la composición urbana. Con el movimiento moderno, según Martí (1991), la tipología edificatoria es la protagonista hasta tal punto que la relación entre la célula habitable y la forma urbana es una realidad solidaria e interdependiente.

El proyecto de transformación urbana de los últimos 30 años, del punto de vista de la estructura urbana, asimila la dicotomía entre la preocupación exclusiva con el paisaje urbano, y por lo tanto con el espacio público en el siglo XIX, y la preocupación fundamental con el espacio privado en la primera mitad del siglo xx.

En términos de ordenación y de composición, se verifica un intento de síntesis entre la preocupación de los siglos xIX y xx. Así, las categorías tradicionalmente empleadas para explicar el tipo de ordenación (ordenaciones abiertas o cerradas) no son suficientes para explicar las opciones de organización de los elementos libres y edificados en el proyecto de transformación urbana de los últimos 30 años. Se utilizan sobretodo lógicas híbridas, entre su naturaleza extro-vertida o intro-vertida.

Las ordenaciones extro-vertidas son aquellas en que la distribución de los elementos edificados sigue las reglas definidas por el soporte. Es decir, el orden según el cual se distribuyen las piezas en el espacio es dictado por la trama o por el perímetro de la manzana. Las ordenaciones intro-vertidas, por el contrario, son las que siguen un orden interno, independiente de la existencia de un trazado de fondo, de modo intrínseco y autónomo al entorno. 


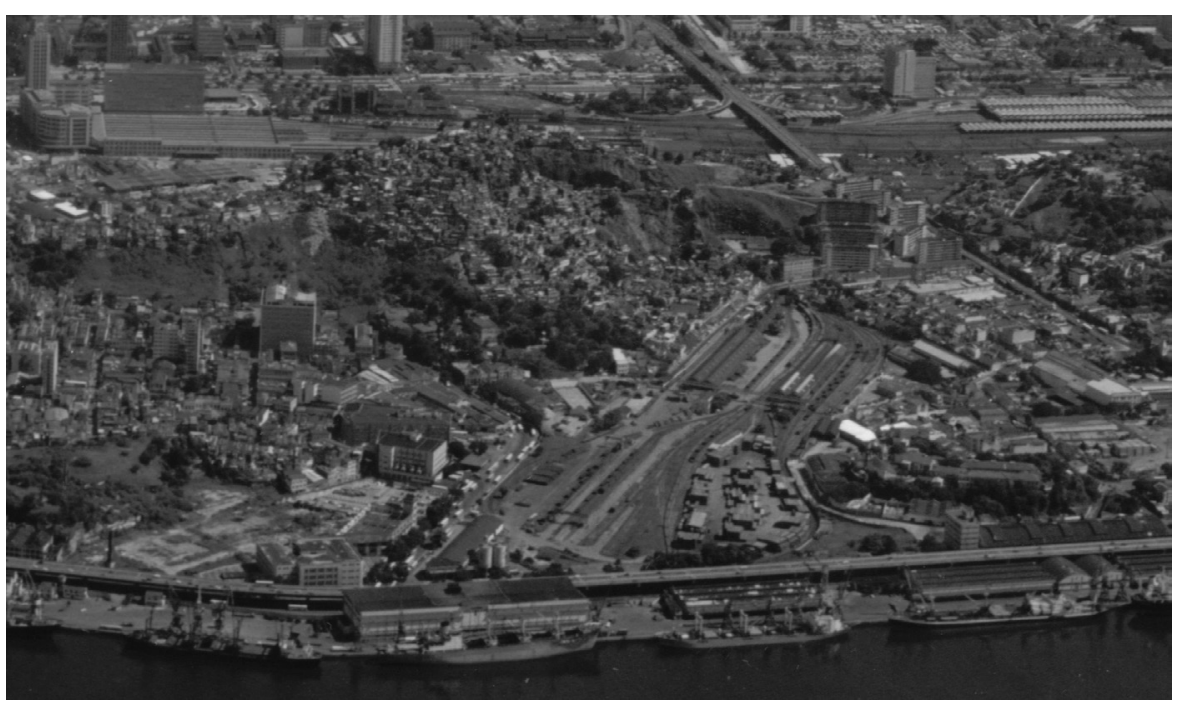

Fig.2- Ordenaciones híbridas. Fuente: MARTINS, Anamaria de Aragão C. Recolonización urbana: nuevos fragmentos urbanos en la transformación de las primeras periferias. 502 p. Tese (Doutorado) - Universitat Politècnica de Catalunya - Escuela Técnica Superior de Arquitectura de Barcelona, Departamento de Urbanismo y Ordenación del Territorio, 2004, disponible en: <http:// www.tesisenxarxa.net/TDX-0627105-085623/ >

Las ordenaciones híbridas, además, oscilan entre los modelos compactos y los disgregados. Estos modelos presentan formas extremas de saturación de la masa construida y del espacio libre respectivamente (Fig. 2).

En Borneo, la solución dada a las unidades urbanas parte de una ordenación extro-vertida con tendencia a la compacidad. La manzana con parcelas individuales adosadas y pareadas es la estrategia para materializar la compacidad. La masa edificada se percibirá continua en cada manzana y el espacio libre es fragmentario, privado e individual. Se adoptan diferentes tipologías con cierta uniformidad para cada manzana. Eventualmente las tipologías adoptadas permiten la percepción de un espacio libre más aglutinado, que, no obstante, se mantiene privado e individual (Fig. 2).

En el caso de la Ensenada de Gamboa, en Río de Janeiro, las edificaciones se distribuyen en los perímetros de los módulos, pero no se agregan. La disgregación de las edificaciones permite que el interior del módulo sea permeable desde el exterior (Fig. 2).

El proyecto urbano de la Villa Olímpica ilustra el tema de las ordenaciones intro-vertidas disgregadas, cuyo efecto es la singularidad morfológica. Si bien la operación tiene una trama como soporte, el proyecto se hace desde el perímetro hacia el interior. Se organiza mediante reglas internas a cada unidad de proyecto, determinadas por diferentes lógicas geométricas empleadas en la distribución de las edificaciones: diagonales, arcos de círculo, 
semi-círculos, además de tipologías especiales para cada volumen edificado (Fig. 2).

\section{La tridimensionalidad del proyecto: composición en estratos}

El paso de la ordenación en planta a la composición tridimensional de la unidad urbana introduce la relación entre planos horizontales y verticales. Se reconoce este paso de lo bidimensional a lo tridimensional como una etapa analítica aunque como proceso proyectual, se suelen construir simultáneamente.

La tridimensionalidad del proyecto de transformación urbana de los últimos 30 años puede ser interpretada como una composición en estratos. Cada estrato está compuesto por ordenaciones que siguen las lógicas híbridas anteriormente descritas.

Se reconocen tres estratos: la planta baja - base que toca el suelo -, el cuerpo representado por las plantas intermedias y el remate configurado por las plantas superiores (Fig. 3).

La planta baja establece las relaciones entre el espacio público y el privado pues, en este nivel, se establecen los accesos principales a la edificación como al interior de la manzana. En este sentido, más que el basamento que sostiene la edificación, se trata de un estrato cuya función es la determinación de los dominios.
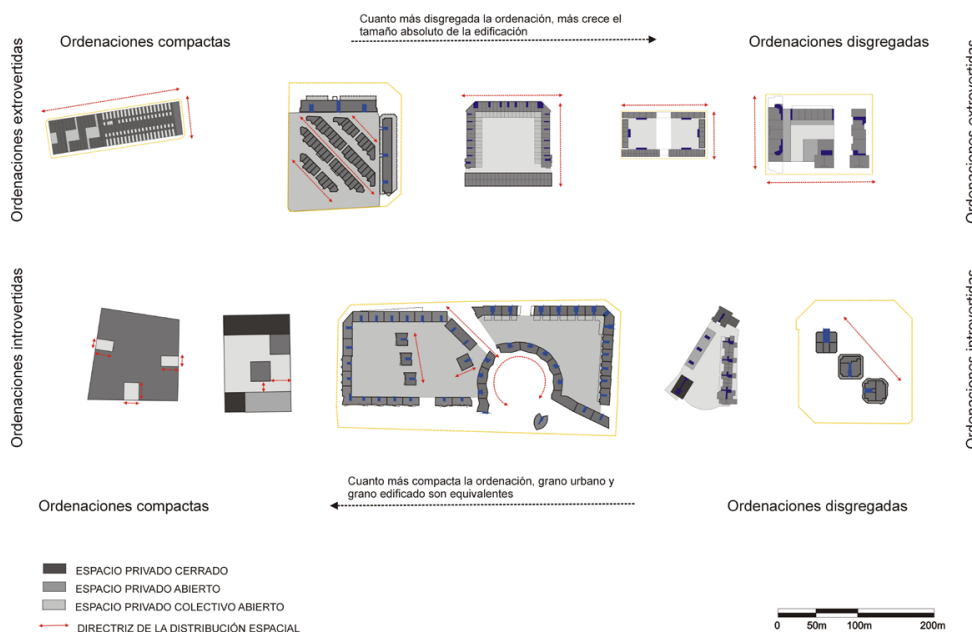

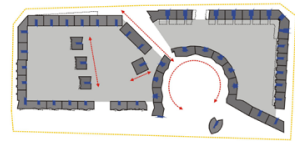

Cuanto más compacta la ordenación. grano urbano y

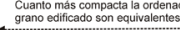

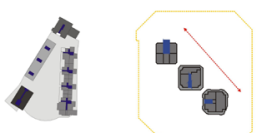

Ordenaciones disgregadas

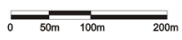

Fig.3- Estratos de la tridimensionalidad. Fuente: MARTINS, Anamaria de Aragão C. Recolonización urbana: nuevos fragmentos urbanos en la transformación de las primeras periferias. 502 p. Tese (Doutorado) - Universitat Politècnica de Catalunya - Escuela Técnica Superior de Arquitectura de Barcelona, Departamento de Urbanismo y Ordenación del Territorio, 2004, disponible en: <http://www.tesisenxarxa.net/TDX-0627105-085623/ > 
La existencia de una planta baja que concentra la masa edificada alineada con los trazados (sistema viario) conforma los límites del espacio público. Por esta razón, la planta baja es un estrato substancial en la configuración del paisaje urbano. No obstante, cuando la planta baja se disgrega en volúmenes fraccionados, la percepción de los límites entre el espacio público y el privado se debilita y ésta pierde su función como referencia del paisaje urbano.

La planta baja que sigue una ordenación extro-vertida pero con tendencia a la disgregación, presenta numerosas interrupciones en la masa edificada perimetral, configurando pasajes. Los pasajes dan acceso a espacios interiores concentrados, con menor posibilidad de movimiento como los patios. El efecto panóptico de "salón" urbano de estos espacios sugiere mayor privacidad y control. Una secuencia de pasajes, por el contrario, determina flujos en una dirección (Fig. 4).

En las plantas bajas de ordenación intro-vertidas con tendencia a la disgregación, el espacio libre de la manzana es el fondo que soporta los volúmenes dispersos. No se asocian a estos espacios libres una clara función en el movimiento o en la configuración del paisaje urbano. Muchas veces, se los denomina jardines o parques. Sin embargo, son espacios residuales, de dominio ambiguo.

En las plantas bajas con ordenación intro-vertida, con tendencia a la compacidad, se observan espacios libres bien delimitados con geometría propia. Plazas en forma circular o elíptica como los "crecents" ingleses, o bien el "square" del siglo xIx reaparecen como espacios libres concentrados en las planta bajas cuyas masas edificadas tienden a agregarse (Fig. 4).

El cuerpo edificado es un estrato con ordenación propia y en gran parte de los casos, diferente de la ordenación de la planta baja. El punto de continuidad son los sistemas de accesos verticales.

Los cuerpos con ordenación extro-vertida tienden a alinearse con los perímetros de la manzana. Este recurso configura una fachada urbana que delimita el espacio público exterior. Este recurso no es novedoso en el sentido de que reproduce las preocupaciones por el paisaje urbano presentes en las composiciones del siglo xIx. La fachada urbana generada por la alineación perimetral de los cuerpos edificados es la principal característica de la ciudad uniforme y regular.

La búsqueda de un modelo intro-vertido de ordenación para los cuerpos edificados huye de la solución regular. Si la regla del cuerpo urbano es interna, el efecto será la singularidad de la manzana y, no, la definición de una fachada uniforme. Como estrategia, se buscan retranqueos en puntos concretos y no de toda la fachada. Se establece una intención rítmica re- 
lacionada con la lógica interna de la unidad urbana. Si además el cuerpo se disgrega, entonces el efecto fragmentario de la masa construida resulta en la autonomía del volumen de la edificación respecto al paisaje urbano.

El remate configurado por las plantas superiores suele depender del tipo de ordenación definida en el nivel del cuerpo edificado.

En las ordenaciones híbridas, se juega con ambas estrategias de regularidad e irregularidad para el último estrato de la manzana. Se observa que aquellos proyectos que optan por cuerpos más disgregados se inclinan por remates solidarizados con el cuerpo edificado. En contraposición, cuerpos menos disgregados, acaban por disociar cuerpo y remate.

Los remates influencian la percepción del skyline en ciertas alturas y mediante ciertas distancias respecto a la fachada. En edificios muy altos, por ejemplo, el cuerpo edificado tendrá más influencia que el remate, mientras en edificios bajos, los remates sí delimitan el skyline.

Composiciones tridimensionales que optan por la disgregación de los elementos edificados tienden a presentar menor posibilidad de variación de cada uno de sus estratos (planta baja, cuerpo y remate). Los elementos disgregados son percibidos como una unidad. La singularidad de la composición se verifica por la regla intro-vertida que la disgregación permite.

El ejemplo de la torre es paradigmático. Una composición con torres es un modelo disgregado e intro-vertido. Si bien se intente variar la planta baja, cuerpo y remate, se percibe la tipología con cierta homogeneidad lo que supera posibles variaciones en los diferentes estratos de la edificación.

En Diagonal Mar, en Barcelona, las edificaciones responden a una lógica interna que configura una unidad reconocida como un módulo: torres articuladas por un bloque bajo. La lógica de la ordenación independe del soporte. La ordenación es disgregada, pues los edificios que componen el módulo no se aglutinan. Se genera un espacio libre interior al módulo que, no obstante, es privado y colectivo (Fig. 5).

En contraposición, las ordenaciones extro-vertidas que agrupan los elementos edificados con cierta continuidad respecto a un soporte agrandan la importancia de la ordenación de cada estrato. La dimensión de conjunto refuerza el sentido de cada estrato, más que la individualización de cada elemento edificado. La planta baja en general tendrá cierta disgregación creando espacios interiores permeables con el exterior. El cuerpo, pese a implicación de las alineaciones, tiene la posibilidad de retranquearse en determinados puntos y de variar las alturas. El remate puede asumir cierto grado de disgregación permitiendo también numerosas variaciones tridimensionales. 


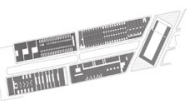

MORFOLOGÍA DEL ÁMBITO DE INTERVENCIÓN

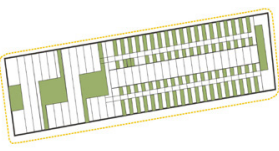

ORDENACIÓN DE UNA UNIDAD URBANA

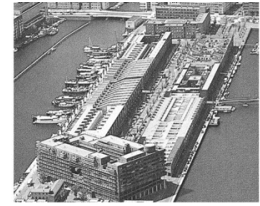

COMPOSICIÓN VOLUMÉTRICA

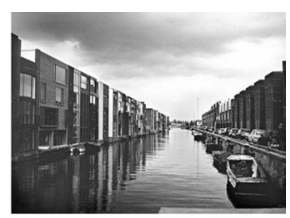

TIPOLOGÍA

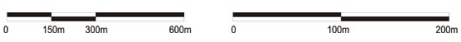

Fig.4- Espacios libres derivados de la ordenación. Fuente: MARTINS, Anamaria de Aragão C. Recolonización urbana: nuevos fragmentos urbanos en la transformación de las primeras periferias. 502 p. Tese (Doutorado) - Universitat Politècnica de Catalunya - Escuela Técnica Superior de Arquitectura de Barcelona, Departamento de Urbanismo y Ordenación del Territorio, 2004, disponible en: <http://www.tesisenxarxa.net/TDX-0627105-085623/ >

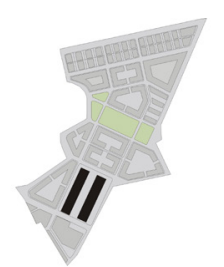

MORFOLOGÍA DEL ÁMBITO DE INTERVENCIÓN
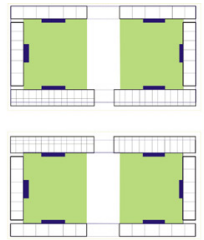

ORDENACIÓN DE UNA UNIDAD URBANA

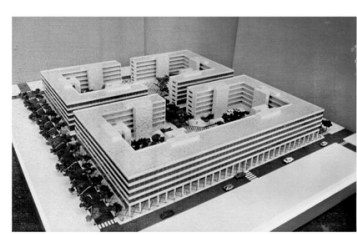

COMPOSICIÓN VOLUMÉTRICA

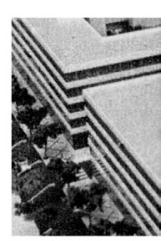

TIPOLOGIA

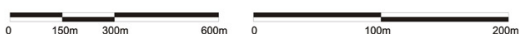

Fig.5- Ordenaciones en Diagonal Mar, Barcelona. Fuente: MARTINS, Anamaria de Aragão C. Recolonización urbana: nuevos fragmentos urbanos en la transformación de las primeras periferias. $502 \mathrm{p}$. Tese (Doutorado) - Universitat Politècnica de Catalunya - Escuela Técnica Superior de Arquitectura de Barcelona, Departamento de Urbanismo y Ordenación del Territorio, 2004, disponible en: <http://www.tesisenxarxa.net/TDX-0627105-085623/>

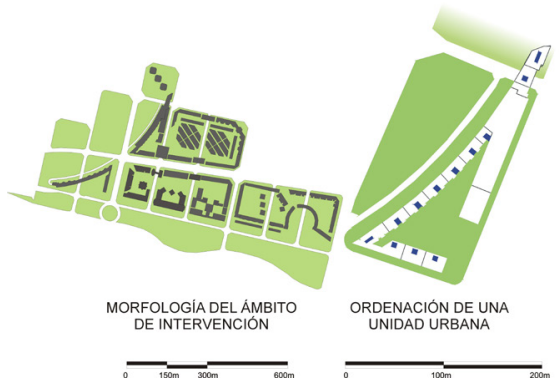

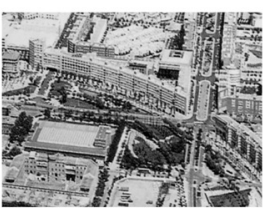

COMPOSICIÓN VOLUMÉTRICA

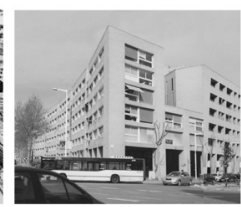

TIPOLOGIA

Fig.6- Ordenaciones en Abattoirterrein, Ámsterdam. Fuente: MARTINS, Anamaria de Aragão C. Recolonización urbana: nuevos fragmentos urbanos en la transformación de las primeras periferias. 502 p. Tese (Doutorado) - Universitat Politècnica de Catalunya - Escuela Técnica Superior de Arquitectura de Barcelona, Departamento de Urbanismo y Ordenación del Territorio, 2004, disponible en: <http://www.tesisenxarxa.net/TDX-0627105-085623/ > 
En el proyecto del Abattoirterrein, en Ámsterdam, la planta baja es un estrato disgregado. Los volúmenes edificados se perciben fragmentarios pese a su alineación con la trama. La disgregación de la planta baja permite que el espacio libre interior se conecte con las áreas públicas. Los cuerpos edificados, en cambio, están alineados con la trama de soporte. Aunque se reconozcan las diferentes edificaciones que forman la unidad urbana, pues son arquitectónicamente diferentes, una misma alineación y altura sugieren la configuración de una fachada urbana regular. Además estos cuerpos edificados se concentran en los perímetros de la manzana, configurando una fachada para los patios interiores. La altura no superior a 8 plantas del cuerpo permite que la ordenación del remate tenga influencia sobre el paisaje (Fig. 6).

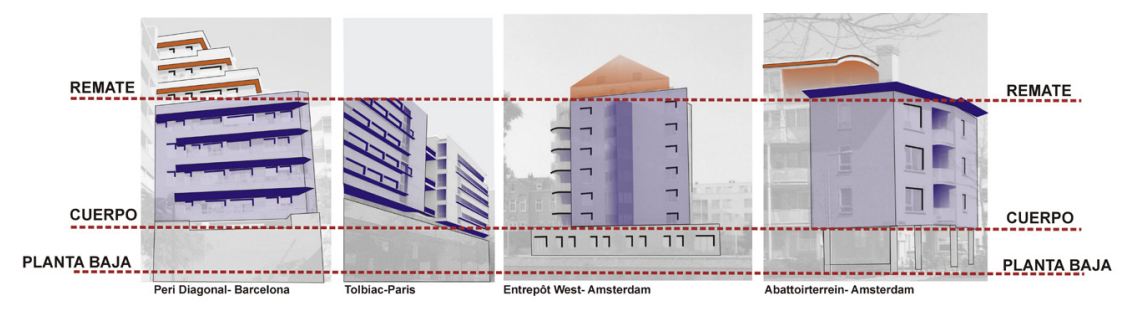

Fig.7- Ordenaciones en la ZAC Masséna, París. Fuente: MARTINS, Anamaria de Aragão C. Recolonización urbana: nuevos fragmentos urbanos en la transformación de las primeras periferias. 502 p. Tese (Doutorado) - Universitat Politècnica de Catalunya - Escuela Técnica Superior de Arquitectura de Barcelona, Departamento de Urbanismo y Ordenación del Territorio, 2004, disponible en: <http://www.tesisenxarxa.net/TDX-0627105-085623/ >

Eso significa que la manzana ordenada de modo extro-vertido con cierto grado de agregación de los elementos edificados permite mayor diversidad en el efecto final de la composición que modelos disgregados, como los basados en edificaciones aisladas. Este grado de diversidad contribuye a las transiciones con el entorno, territorio igualmente heterogéneo.

\section{Buscar coherencia entre el nuevo y el existente}

Los proyectos de transformación urbana deben afrontar el problema del encaje de una nueva morfología en la diversidad de la ciudad tradicional, $y$ en especial, en la heterogeneidad de las periferias.

Como la ciudad tradicional se construye parcela a parcela, reglas disímiles se perciben en este nivel, como resultado de las diferentes ordenanzas, así como por las mutaciones que suelen acontecer a lo largo del tiempo en el espacio urbano.

En la transformación urbana, sin embargo, no hay espacio a la "natural" diversidad. Ya no se construye parcela a parcela. Cada incorporador gestiona piezas urbanas y definen la forma urbana en un período de tiempo menos extendido. 
Frente a esa forma de gestión de las operaciones, la transformación urbana de los últimos 30 ańos busca estrategias de proyecto que permitan simular la diversidad, mediante algunos recursos de diseño en el nivel de la composición tridimensional de las unidades urbanas.

Una estrategia se refiere a la contribución de diferentes arquitectos sobre una misma unidad operativa. En algunos casos, como la Villa Olímpica, en Barcelona, o el proyecto de Borneo, en Ámsterdam, se eligen puntos estratégicos para el cambio de arquitecto - la esquina, por ejemplo.

Por otra parte, algunas estrategias intentan simular la diversidad que se observa con la variación de ordenanzas a lo largo del tiempo. Son reglas que configuran la ordenación dinámica, como en el caso de Masséna, en Paris.

Por una parte, el proyecto define como elemento invariable los zócalos de igual altura, ordenado según el soporte. En el nivel del cuerpo edificado, el proyecto demuestra preocupación con la estructura al definir que la variación de la altura de las edificaciones depende de la anchura de las calles de la trama de planta baja. Se trata de establecer una relación entre los elementos del orden global del proyecto y de la ordenación específica de cada manzana. La fuerza de la lógica intro-vertida para los cuerpos edificados radica en el encadenamiento de las reglas de edificación, que aportan dinamismo al resultado final de la composición tridimensional.

Como reglas generales, se define que la edificabilidad de las manzanas se limita por un valor constante de $40 \%$ a partir de $15 \mathrm{~m}$ de altura que, sin embargo, no será dividida de manera homogénea entre las parcelas. La primera parcela edificada condicionará todas las demás que tendrán de compartir la edificabilidad que resta. En el caso de implantación de edificios bajos, el potencial constructivo podrá ser transferido a la parcela vecina, que podrá absorberlo en una mayor altura de la edificación. La regla de composición es, por tanto, interna a cada manzana y variable según la secuencia de edificación (Fig. 7).

El efecto final de esta estrategia, basada en la diversidad de alturas y alineaciones, se asemeja a las mutaciones lo largo del tiempo, como en los procesos de transformación tradicionales.

\section{Aportar diversidad como una estrategia de proyecto}

Utilizar la diversidad para un mejor encaje entre lo nuevo y lo existente depende del grado de heterogeneidad del entorno y del nivel en qué está presente (si en el grano urbano o edificado). Tridimensionalmente, el proyecto debe buscar comprender en cual estrato radica la diversidad del territorio en que se inserta. Eso orientará, por ejemplo, decisiones por 
plantas bajas más disgregadas y cuerpos más agregados o, al revés, definir bases agregadas con la variación en los cuerpos de las edificaciones.

En el caso de que se establezcan reglas relativamente uniformes para la globalidad de las unidades urbanas, la diversidad podría ser buscada en la multiplicidad de las arquitecturas. Eso implica adoptar un tipo de lógica de gestión de la intervención aproximada a las lógicas de la ciudad tradicional, con propiedades fragmentarias e incorporadores diversos. La unidad urbana tiene que presentar un grano edilicio de menor dimensión para que la diversidad se limite a la escala arquitectónica.

Si se opta por preconfigurar la diversidad de la manzana, dos estrategias pueden adoptarse. Una es especificar el tipo de ordenación y composición, la distribución de cada nivel de la unidad urbana (planta baja, cuerpo y remate) según un orden extro-vertido o intro-vertido, con tendencia a la compacidad o a la disgregación. Son figuraciones que obligan el detalle de todas y cada una de las unidades urbanas. Otra estrategia es establecer reglas abstractas pero encadenadas, en que la ocupación de cada parcela condiciona la forma urbana de las demás. La diversidad en este caso se obtiene de una secuencia de decisiones tomadas a lo largo del desarrollo de la intervención.

Como una de las conclusiones del trabajo, se observa que la relación que permite el encaje de un nuevo tejido en una situación heterogénea, como la de las primeras periferias, se refiere a un grado intermedio de diversidad que se manifiesta en la textura del proyecto urbano.

Composiciones tridimensionales demasiado uniformes anulan la percepción de la edificación. Predominará la percepción de la masa edificada de la manzana como un elemento "sólido" y homogéneo. Si el entorno no sigue las mismas reglas compositivas de esta unidad uniforme, entonces el contraste entre ambas configuraciones será contundente. La regla opuesta tampoco garantiza mucha coherencia. Una composición demasiado singular individualiza el edificio, de modo que tampoco se observan las características de repetición y cierta continuidad necesarias a la percepción de un tejido cohesionado. Frente a la heterogeneidad de las primeras periferias, la búsqueda de cierto grado de diversidad para la composición de la manzana es una estrategia interesante para generar transiciones con mayor continuidad.

El efecto de las ordenaciones y composiciones híbridas del proyecto de transformación urbana de los últimos 30 años favorece el encaje en su contexto. La diversidad, asociada a otras propiedades de diseño urbano, como la definición de una trama articuladora y de espacios libres vertebradores, contribuye en la capacidad del nuevo fragmento urbano en dialogar con el 
territorio en que se inserta, definiendo el proceso de transformación urbana como una estrategia positiva de integración de las periferias industriales con la ciudad central.

\section{Referencias bibliográficas.}

DiPASQUALE, D. \& WHEATON, W. Urban Economics and Real Estate Markets. New Jersey: Prentice Hall, 1996.

MARTí, C (org). Las formas de la residencia en la ciudad moderna. Barcelona: Ed. UPC, 1991.

MARTINS, Anamaria de Aragão C. Recolonización urbana: nuevos fragmentos urbanos en la transformación de las primeras periferias. 502 p. Tese (Doutorado) - Universitat Politècnica de Catalunya - Escuela Técnica Superior de Arquitectura de Barcelona, Departamento de Urbanismo y Ordenación del Territorio, 2004, disponible en:<http://www.tesisenxarxa.net/TDX-0627105-085623/ >

MARTINS, Anamaria de Aragão C. Transformação urbana: projetando novos bairros em antigas periferias. Brasília: Thesaurus, 2012.

Anamaria de Aragão Costa Martins, arquitecta. Doctora en Urbanismo (UPC)

Urbanista del Gobierno del Distrito Federal Profesora del Centro de Ensino Universitário de Brasília - UNICEUB. anamaria.martins@uniceub.br 